Research Paper

\title{
High PD-L1 Expression Is Closely Associated With Tumor-Infiltrating Lymphocytes and Leads to Good Clinical Outcomes in Chinese Triple Negative Breast Cancer Patients
}

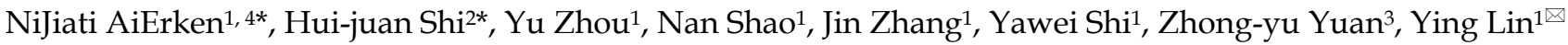 \\ 1. Breast Disease Center, The First Affiliated Hospital, Sun Yat-sen University, Guangzhou 510080, China; \\ 2. Department of Pathology, The First Affiliated Hospital, Sun Yat-sen University, Guangzhou 510080, China; \\ 3. Department of Medical Oncology, Sun Yat-sen University Cancer Center, Guangzhou 510060, China; \\ 4. Department of Breast and Thyroid Surgery, The Seventh Affiliated Hospital, Sun Yat-sen University, ShenZhen, 518107, China. \\ * These two authors contributed equally to this work. \\ $\square$ Corresponding author: linying3@mail.sysu.edu.cn, phone number: (+86) 13076873871 \\ (c) Ivyspring International Publisher. This is an open access article distributed under the terms of the Creative Commons Attribution (CC BY-NC) license \\ (https://creativecommons.org/licenses/by-nc/4.0/). See http://ivyspring.com/terms for full terms and conditions.
}

Received: 2017.05.04; Accepted: 2017.07.11; Published: 2017.09.05

\begin{abstract}
Background: To investigate the role of Programmed death ligand 1 (PD-LI) expression and tumor-infiltrating lymphocytes (TILs) in tumor recurrence and metastasis of Chinese patients suffering from triple negative breast cancer (TNBC).

Methods: PD-L1 immunohistochemistry was performed on 215 TNBCs. Also, the prevalence of TILs correlated the expression of PD-L1 and TILs with clinical outcomes. Kaplan-Meier and the model analyses of univariate Cox proportional hazards were utilized to compare the survival of patients with positive PD-L1 expression with those with negative PD-L1 expression.

Results: The median follow-up time was 67.7 months (range: 7-159 months). PD-L1-positive breast cancer patients had significantly longer disease-free survival (DFS) and Overall survival (OS) compared with PD-LI-negative patients $(P=0.046 ; P=0.019)$ in TNBC. The presence of increased stromal lymphocytic infiltrates (STILs) was significantly associated with overall survival $(P=0.026)$. The model analysis of univariate Cox proportional hazards showed that PD-LI and STILs were independent prognostic factors for tumor prognosis.

Conclusions: Our study found that high levels of PD-L1 could be expressed in TNBC, which was correlated with the prevalence of TILs.
\end{abstract}

Key words: PD-L1, TIL, breast cancer, TNBC, prognosis.

\section{Introduction}

The 2015 ACS cancer statistics released by Breast Cancer Account of United States showed that the incidence of breast cancer amongst women (25\%) was the first primary of the total incidence of cancer in the Unites States. Particularly, the mortality of female breast cancer had reached 40,000 , accounting for $15 \%$ of the total mortality [1]. By comparing with the data in 2008, the World Cancer Statistics in 2012 showed that the mortality of Chinese breast cancer had dramatically increased from 48,000 to 697,000 [2]

Despite the progress in increasing the therapeutic regimen for breast cancer, within a decade, distant metastasis or recurrence have occurred to more than $50 \%$ patients with invasive breast cancer, resulting in treatment failure [3]. In fact, a 5-year survival for 'triple-negative breast cancer' (TNBC) was only $60 \%$ [4]. Thus, it is essential to introduce an innovative treatment approach to the 
specific patient group.

Researches have paid more attention to the function of immunoregulatory receptor programmed cell death 1 (PD-1) and the corresponding B7 family of ligands as a pivotal mechanism of tumor immune tolerance and its escape in cancer. $\mathrm{T}$ cell can be activated by two main signal pathways. One way refers to the TCR receptors on the surface of the T cell discern antigen-presenting cells through the expression of MHC molecules that present the antigen peptide. Another is a way through the antigen presenting cells expression of activated molecules with $\mathrm{T}$ cell surface receptors to produce the corresponding stimulation signal. Lacking or rejection of stimulus signal would lead to T cells' inactivation [5-7]. In addition to the positive activated molecules, the negative stimulus molecule also widely exists in the immune regulation process which has inhibition function for $\mathrm{T}$ cell. PD-L1, standing for the programmed death ligand 1, is an important member of immunoglobulin superfamily negative stimulus molecule to participate in the immune regulation process. PD - L1 is the main ligand of Programmed death receptor 1 (PD - 1). PD-L1 has been found by Ishida el. in an apoptosis research of $\mathrm{T}$ cell hybridoma cell apoptosis related proteins [8]. Also, the PD - L1 signaling pathways can suppress the negative regulation of $\mathrm{T}$ cell immune response and the immune tolerance for microbial infection and tumor.

It has been approved that there is an association between tumor-infiltrating lymphocytes (TILs) express and high PD-L1expression in varieties of cancers [9-14]. Researchers found that CTLA4 expression is associated with the development of breast cancer and PD-L1+TILs are also related to prognosis [15].With the advances of technology, immune-targeting drugs have been approved to be applied in lung cancer and melanoma clinical applications [16, 17]. However, the PD-L1 expression and prevalence of tumor TILs in Chinese TNBC patients still remain unclear. Today, very limited data on the expression of PD-L1 in Chinese TNBC patients have been reported.

This study aimed to search for the relationship among the expression level of PD-L1, TILs and clinical manifestations in Chinese patients with triple negative breast cancer, particularly in the aspects of prognostic value.

\section{Materials and Methods}

\section{Breast cancer Tissue Microarray (TMA) and immunohistochemistry}

31 Breast Cancer Tissue was acquired from the patients in the First Affiliated Hospital of Sun Yat-sen
University and 184 Breast Cancer Tissue was acquired from patients in Sun Yat-sen University cancer center, who had undergone surgical resection and had been diagnosed with TNBC. 215 samples (including recurrent and metastatic) from July1999 to October 2012 were admitted by the cancer center review board of the First Affiliated Hospital of Sun Yat-sen University and Sun Yat-sen University. TNBC patients were identified for the tissue microarray (TMA) immunohistochemical staining. Patient's clinical information was collected, including age, gender, tumor location(s), disease status of breast cancer, lymph node involvement and ER, PR and HER2 status. Supplementary information including adjuvant chemotherapy and radiotherapy was collected as well.

Slides were stained based on the Immunohistochemistry Protocol (Paraffin) from Cell Signaling Technology (Beverly, MA). Briefly, 5 - $\mu$ m-thick array sections were baked at $65^{\circ} \mathrm{C}$ for $3 \mathrm{~h}$, dewaxed with xylene (two times for 5 minutes each), putted through $100 \%$ ethanol (twice for 5 minutes each) and rehydrated through various graded alcohol. Subsequently, the slide was washed with PBS for three times for 5 minutes. Antigen retrieval was coped with Target Retrieval Solution (Dako, North America, Inc., CA). After the antigen retrieval, the slide was rinsed with PBS for three times for 5 minutes. Following the process of antigen retrieval, endogenous peroxidase activity was quenched by incubation in 3\% hydrogen peroxide, and the protein was blocked with blocking solution (Cell Signaling Technology) for 10 minutes at room temperature. The concentration of rabbit primary antibody which reacted to PD-L1 (Cell Signaling Technology, Beverly, MA) was 1:100 in Dako antibody diluent; slides were applied at $4{ }^{\circ} \mathrm{C}$ overnight. Afterwards, the slides were incubated with Ventana Omni Mapanti-rabbit secondary antibody for 60 Minutes. Each step was succeeded by three Tris-buffered saline (TBS) rinses, and the bound antibody on the array was detected by utilizing SignalStain ${ }^{\circledR}$ Boost Detection Reagent (Cell Signaling Technology) and SignalStain ${ }^{\circledR}$ DAB (Cell Signaling Technology). Finally, sections were counterstained with Hematoxylin QS (VectorLaboratories) and the slide was mounted with VectaMount AQ (Vector Laboratories) for long-term preservation. Two independent pathologists had checked all the slides. Both of them had not known the patient's clinical parameters, which eliminated the observation biases.

The immunostaining intensity pattern of PD-L1 was assessed based on a scale semi-quantitatively as follows: (1) intensity of the stain: 0 , no staining; $1+$, weak staining; $2+$, moderate staining; and $3+$, intense 
staining. (2) positive cell percentage rate: 0 , cells $<5 \%$, $1,5 \% \sim 25 \%, 2,26 \% \sim 50 \%, 3,51 \% \sim 75 \%, 4,>75 \%$. Scoring was calculated from the mean of the two independently conducted researchers. After the calculation, overall result of staining intensity was recorded as: negative $(\leq 1)$ and positive $(>1)$. PD-L1 staining images were obtained by utilizing a Nikon Eclipse Ti-U fluorescence microscope (Nikon Corp) with a SPOTRT digital camera (Diagnostic Instruments Inc).

\section{Evaluation of tumor-infiltrating lymphocytes (TIL)}

TILs were separately scored by two independent pathologists. On the basis of the prespecified analysis plan, the presence of TILs was evaluated by the 200×magnification and semi-quantitatively as below: score 1, TILs-low (range, $0 \%$ to $10 \%$ ); 2, TILs-moderate (range, $11 \%$ to $40 \%$ ); 3, TILs-Marked (range, $41 \%$ to $100 \%)$.

\section{Statistical analysis}

All the statistical analyses were performed utilizing the SPSS statistics software version 19. Kaplan-Meier survival curves were generated to examine the relationship between the expression levels of PD-L1 and the survival rate of patients. Overall survival (OS) was calculated from the date of tumor diagnosis to the date of death from any cause, or time of the last visit. The primary endpoint for this analysis was disease-free survival (DFS) which can be defined as the length of time from the date of tumor diagnosis to local, regional, or distant recurrence or to the date of death from any cause. The statistical significance between the two groups was determined by the usage of chi-square test, or two-sample t-test as appropriate. The statistical significance was described in figures and legends. The significance was set at a p-value of 0.05 .

\section{Results}

\section{TILs and PD-LI expression in TNBC tissue}

Patient characteristics: 215 Chinese patients with TNBC in total were enrolled in our study. The median age at diagnosis was 49.0 years (range: $27-78$ years). The characteristics of these patients are listed in Table 1 , as shown in which, we collected clinicopathological parameters including median age, stage surgical methods, positive lymph nodes, tumor size, chemotherapy, radiotherapy, neo-adjuvant chemotherapy, Stromal TIL, Intra-tumoral TIL and total TIL. Associations could be found between TNBC PD-L1 expression and STILs, LPBC as well as surgical approaches.
Table 1. PD-LI expression levels of 215 TNBC

\begin{tabular}{|c|c|c|c|c|c|}
\hline \multirow[t]{2}{*}{ variable } & & \multicolumn{2}{|l|}{ PD-L1 } & \multirow{2}{*}{$-\mathrm{X}^{2}$ value } & \multirow{2}{*}{$\begin{array}{l}P \\
\text { value }\end{array}$} \\
\hline & & $\begin{array}{l}\text { negative } \\
\mathrm{n}=145\end{array}$ & $\begin{array}{l}\text { positive } \\
\mathrm{n}=70\end{array}$ & & \\
\hline $\begin{array}{l}\text { Median } \\
\text { age(years) }\end{array}$ & & $49(27-78)$ & & & \\
\hline \multirow[t]{4}{*}{$\begin{array}{l}\text { Tumor } \\
\text { size(mm) }\end{array}$} & & & & 2.143 & 0.260 \\
\hline & $<20$ & $33(15.3)$ & $14(6.5)$ & & \\
\hline & $20-50$ & $95(44.2)$ & $54(25.1)$ & & \\
\hline & $\geq 50$ & $17(7.9)$ & $2(0.9)$ & & \\
\hline \multirow[t]{5}{*}{$\begin{array}{l}\text { Positive } \\
\text { Lymph nodes }\end{array}$} & & & & 8.260 & 0.313 \\
\hline & 0 & $79(36.9)$ & $43(20.1)$ & & \\
\hline & $1-3$ & $40(18.7)$ & $12(5.6)$ & & \\
\hline & $4-9$ & $11(5.1)$ & $12(5.6)$ & & \\
\hline & $\geq 10$ & $14(6.5)$ & $3(1.4)$ & & \\
\hline \multirow{3}{*}{$\begin{array}{l}\text { Surgical } \\
\text { methods }\end{array}$} & & & & 4.535 & 0.027 \\
\hline & Radical mastectomy & $136(63.3)$ & $70(32.6)$ & & \\
\hline & Conservative surgery & $9(4.2)$ & $0(0)$ & & \\
\hline \multirow[t]{4}{*}{ Stage } & & & & 0.278 & 0.350 \\
\hline & I & $23(10.7)$ & $10(4.7)$ & & \\
\hline & II & $95(44.2)$ & $45(20.9)$ & & \\
\hline & III & $27(12.6)$ & $15(7.0)$ & & \\
\hline \multirow[t]{5}{*}{ Chemotherapy } & & & & 0.629 & 0.890 \\
\hline & No Chemotherapy & $12(5.6)$ & $5(2.3)$ & & \\
\hline & Anthracyclines & $45(20.9)$ & $24(11.2)$ & & \\
\hline & Anthracyclines+Taxinol & $62(28.8)$ & $31(14.4)$ & & \\
\hline & Other & $26(12.1)$ & $10(4.7)$ & & \\
\hline \multirow[t]{3}{*}{ Radiotherapy } & & & & 0.844 & 0.230 \\
\hline & No & $110(51.2)$ & $57(26.5)$ & & \\
\hline & Yes & $35(16.3)$ & $13(6.0)$ & & \\
\hline \multirow[t]{3}{*}{$\begin{array}{l}\text { Neo-adjuvant } \\
\text { chemotherapy }\end{array}$} & & & & 0.685 & 0.296 \\
\hline & No & $132(61.4)$ & $66(30.7)$ & & \\
\hline & Yes & $13(6.0)$ & $4(1.9)$ & & \\
\hline \multirow[t]{4}{*}{ Stromal TIL } & & & & 4.170 & 0.027 \\
\hline & $0-10$ & $90(41.9)$ & $34(15.8)$ & & \\
\hline & $10-40$ & $35(16.3)$ & $20(9.3)$ & & \\
\hline & $40-100$ & $20(9.3)$ & $16(7.4)$ & & \\
\hline \multirow[t]{4}{*}{$\begin{array}{l}\text { Intra-tumoral } \\
\text { TIL }\end{array}$} & & & & 4.731 & 0.259 \\
\hline & $0-10$ & $138(64.2)$ & $63(29.3)$ & & \\
\hline & $10-40$ & $5(2.3)$ & $7(3.3)$ & & \\
\hline & $40-100$ & $2(0.9)$ & $0(0)$ & & \\
\hline \multirow[t]{4}{*}{ TOTAL TIL } & & & & 4.681 & 0.037 \\
\hline & $0-10$ & $79(36.9)$ & $32(15.0)$ & & \\
\hline & $10-40$ & $42(18.0)$ & $18(8.4)$ & & \\
\hline & $40-100$ & $23(10.7)$ & $20(9.3)$ & & \\
\hline \multirow[t]{3}{*}{ Stromal TIL } & & & & 6.406 & 0.013 \\
\hline & $<50$ & $136(63.3)$ & $58(27.0)$ & & \\
\hline & $\geq 50(\mathrm{LPBC})$ & $9(4.2)$ & $12(5.6)$ & & \\
\hline
\end{tabular}

Abbreviations: Programmed death ligand 1 (PD-L1), Few stromal lymphocytic infiltrates (STILs) (0-10\%), Moderate STILs (11\%-40\%), Marked STILs (41\%-100\%); Few Intra-tumoral TIL (ITILs) (0-10\%), Moderate ITILs $(11 \%-40 \%)$, Marked ITILs $(41 \%-100 \%)$. Few TOTAL TILs $(0-10 \%)$, Moderate TOTAL TILs $(11 \%-40 \%)$, Marked TOTAL TILs (41\%-100\%); $\geq 50 \%$ STILs classified as "lymphocyte predominant breast cancer" (LPBC)

PD-L1 was found in the cytoplasm or at the membrane (or both) of tumor cells by immunohistochemical staining (Figure 1). Intratumor PD-L1 expression was observed in $70(32.6 \%)$ patients. Lymphocytic infiltration was observed in most of the tumors. As shown in Figure 1, representative pictures of TNBC cases showed different levels of stromal lymphocytic infiltrates (STILs). Based on the scoring 
criteria of TILs in the tumor samples, STILs-few patients (range: 0 to $10 \%$ ) were found in $57.7 \%$ of the cases (124/215). The number of cases with STILs-moderate patients (range: $11 \%$ to $40 \%$ ) was $25.6 \%$ of the samples $(55 / 215)$. The percentage of cases with STILs-marked patients (range: $41 \%$ to $100 \%)$ was $16.7 \%(36 / 215)$. Few Intra-tumoral TIL (ITILs) patients (range: 0 to10\%) were found in $93.5 \%$ of the cases (201/215); ITILs-moderate patients (range: $11 \%$ to $40 \%$ ) were $5.6 \%$ of the samples (12/215); ITILs-marked patients (range: $41 \%$ to $100 \%$ ) were $\quad 0.9 \% \quad(2 / 215)$. Lymphocytepredominant breast cancer (LPBC) involved $\geq 50 \%$. LPBC ( $\geq 50 \%$ STILs) was seen in only $9.8 \%$ of TNBCs (21of 215).

\section{Relation between PD-LI expression and prognosis}

The median follow-up time was 67.7 months (range: 7-159 months). The PD-L1-positive breast cancer patients had significantly longer DFS and OS values than those of PD-L1-negative patients $(\mathrm{P}=0.046 ; \mathrm{P}=0.019$; Figure 3).

\section{Relation between TILs expression and prognosis}

The presence of increased STILs was significantly associated with survival. STILs-few patients exhibited significantly lower median overall survival rate compared with STILs-moderate and STILs-marked patients $(p=0.026)$. The overall survival of STILs-few patients was considerably poorer than that of STILs-moderate and STILs-marked patients (Figure 3). The DFS exhibited a borderline trend in STIL-few patients in comparison to STILs-moderate and STILs-marked patients. However, it did not achieve statistical significance. $(\mathrm{P}=0.325$; Figure 3$)$

\section{Associations between the expression of PD-LI and TILs}

The representative pictures of TNBC cases which showed different levels of TILs were depicted in Figure 2 (A, B, C). The representative pictures of PD-L1 expression were shown in Figure 1 (A, B, C). Expressions of PD-L1 and TILs in TNBC tissues was described above. The STILs scores were found to be in a significant relationship with the PD-L1 expression patients $(\mathrm{P}=0.027)$. However, there was no strong association between ITILs and PD-L1. $(\mathrm{P}=0.259)$. The correlation between mean percentage LPBC and PD-L1 assessments was $0.013(\mathrm{P}<0.05$, Table 2). The PD-L1 protein expression was significantly related to the presence of elevated STILs. Cases with elevated STILs was presented with significantly higher expression levels of PD-L1 (Figure 4, P $=0.004$, Figure 5).

\section{PD-L1 inTNBC tumor cells staining}
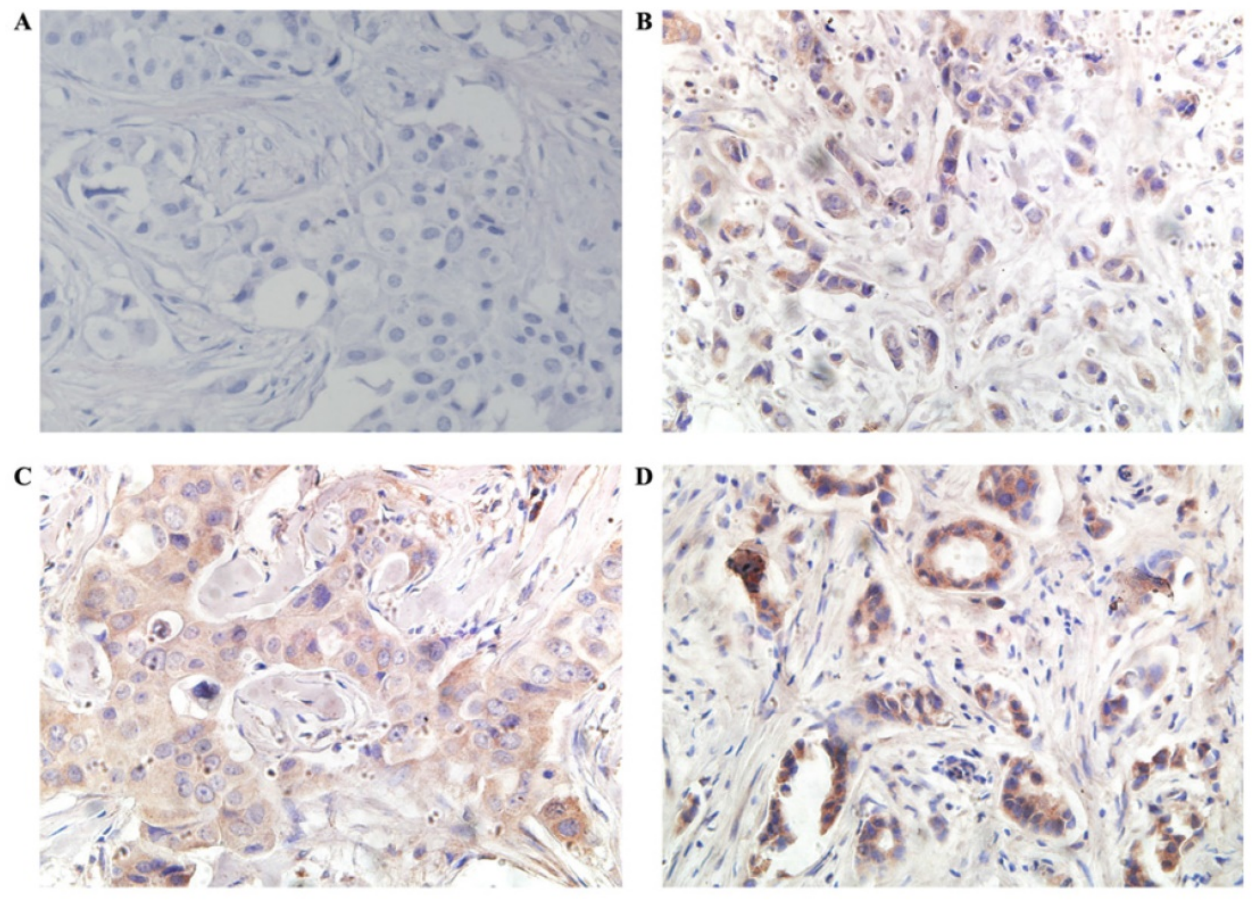

Figure 1. Representative microphotographs of sections from the triple-negative breast cancer samples are showing different tumor infiltrating lymphocyte (TIL) categories and cellular PD-L1 expression compartments. Programmed death ligand 1 (PD-L1) protein $0+(A), 1+(B), 2+(C)$ and $3+(D) .(400 \times)$ 


\section{Levels of TILs}
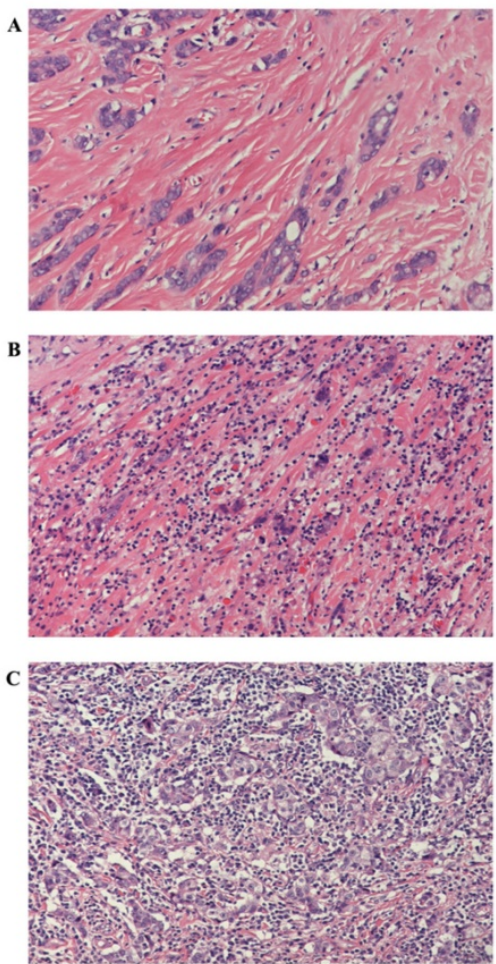

Figure 2. Representative microphotographs of sections from the triple-negative breast cancer samples are showing different tumor infiltrating lymphocyte (TIL) categories: Haematoxylin and eosin-prepared samples showed: few $(0 \%-10 \%)(A)$, moderate (11\%-40\%) (B) and marked (41\%-100\%) (C) TILs.(200x)

\section{Multivariable Analysis}

Upon univariate analysis, only STILs, Total TILs, Tumor size, Positive Lymph nodes and Stage were associated with death from all causes. Multivariable analysis including prognostic variables in both trials confirmed PD-L1and Positive Lymph nodes to be independent prognostic factors for DFS and OS and PD-L1and STILs to be independent prognostic factors for OS (Table 3).

\section{Discussion}

We reviewed the popularity and significance of PD-L1 expression in TNBC. In the study of 215 TNBC patients, the total positivity of PD-L1 was greater than $32.5 \%$. The PD-L1 high expression was associated with STILs, LPBC and surgical methods. However, the relationship between PD-L1 expression and surgical type is questionable as no conservative surgery was performed on PD-L1 positive patients in our study, which could potentially induce an incorrect result, hence more patients should be enrolled to fully assess the connection in future studies. PD-L1 expression was particularly higher in marked-STILs patients. As our data showed, patients with negative PD-L1 expression had significantly decreased their OS and DFS in comparison to those with PD-L1 expression. Multivariable analysis had indicated that PD-L1 expression was a strong independent prognostic factor for patient prognosis and LPBC was an independent prognostic factor for OS.

\section{Prognostic value of PD-L1 expression and TILs expression}
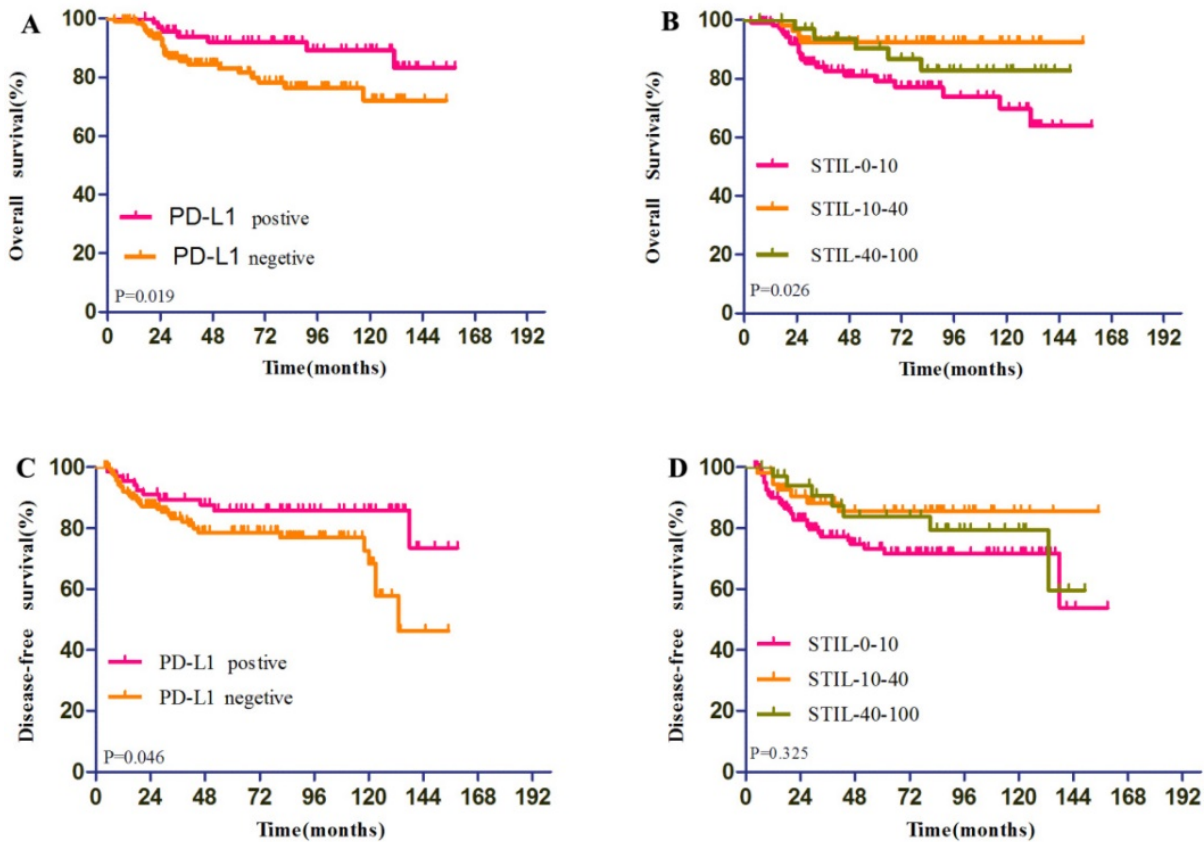

Figure 3. Survival analysis according to Programmed death ligand 1 (PD-LI) expression (A,OS;C,DFS) and stromal lymphocytic infiltrates (STILs) expression (B,OS;D,DFS) 
Table 2. Distribution of TIL Scores

\begin{tabular}{lllllll}
\hline Score $(\%)$ & \multicolumn{2}{l}{ Cancers With STILs } & \multicolumn{2}{l}{ Cancers With ITILs } & \multicolumn{2}{l}{ Total TIL } \\
\cline { 2 - 7 } & No. & $\%$ & No. & $\%$ & No. & $\%$ \\
\hline $0-10$ & 124 & 57.7 & 201 & 93.5 & 111 & 51.9 \\
$11-40$ & 55 & 25.6 & 12 & 5.6 & 60 & 28 \\
$41-100$ & 35 & 16.7 & 2 & 0.9 & 43 & 20.1 \\
$<50$ & 194 & 90.2 & 214 & 99.5 & 183 & 85.5 \\
$\geq 50$ & 21 & 9.8 & 1 & 0.5 & 31 & 14.5 \\
\hline
\end{tabular}

No. of patient cases were in intratumoral and stromal compartments of study and combined with the percentages of tumors. Abbreviations: ITIL, intraepithelial tumor-infiltrating lymphocyte; STIL, stromal tumor-infiltrating lymphocyte; Total TIL, tumor-infiltrating, lymphocyte, lymphocyte-predominant breast cancer (LPBC) which involved $\geq 50 \%$

The experimental result showed further evidence for a biologically significant relation between TNBC and TILs. Recent research on a breast cancer clinical trial provided evidence of TILs in breast cancer and prognostic. The TNBC samples of 481 patients from Eastern Cooperative Oncology Group (ECOG) trials E2197 and E1199 were objective to appraise and investigate the density of stromal compartments and intra-epithelial TILs. Results showed that higher STIL scores were associated with better prognosis. Multivariable analysis confirmed STILs to be an independent prognostic marker of DFS,
DRFI, and OS [18]. Our results were consistent with the TILs results of this report. Anyway, immunity played a significant role in the outcome of primary TNBC.

Associations between the expression of PD-L1 and TILs

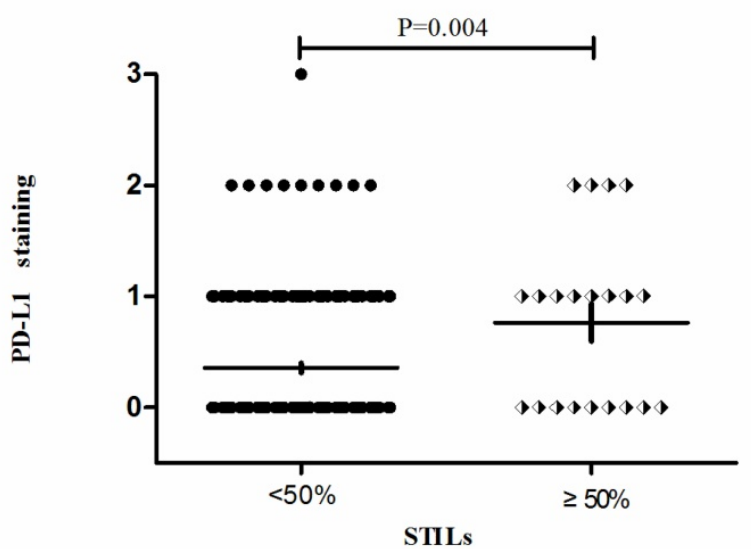

Figure 4. Programmed death ligand 1(PD-L1) protein expression was significantly related to the presence of elevated stromal lymphocytic infiltrates (STILs) and lymphocyte-predominant breast cancer (LPBC) which involved $\geq 50 \%$.

\section{Associations between the expression of PD-L1 and TILs}
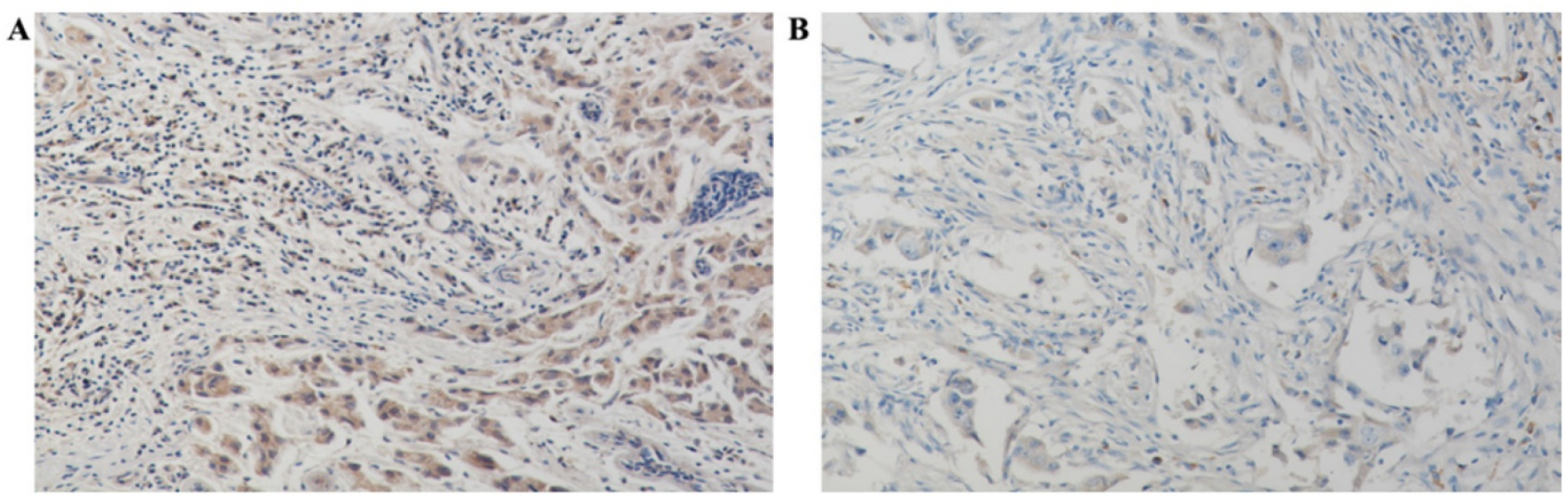

Figure 5. Stromal lymphocytic infiltrates (STILs) presented significantly higher expression levels of Programmed death ligand 1(PD-L1), A: PD-L12+, STILs $\geq 50 \%, B$ : PD-LI1+, STILs<50\%.(200x)

Table 3. Multivariate analyses for OS and DFS

\begin{tabular}{|c|c|c|c|c|c|c|}
\hline \multirow[t]{2}{*}{ Variable } & \multicolumn{3}{|l|}{ OS } & \multicolumn{3}{|l|}{ DFS } \\
\hline & HR & $95 \% \mathrm{CI}$ & $\mathrm{P}$ & HR & $95 \% \mathrm{CI}$ & $\mathrm{P}$ \\
\hline Tumor size $(<2.0 \mathrm{v} 2.1-5.0 \mathrm{v}>5 \mathrm{~cm})$ & 1.615 & 0.868 to 3.004 & 0.13 & 1.763 & 0.936 to 3.323 & 0.079 \\
\hline Grade ( I v II v III) & 0.587 & 0.114 to 3.031 & 0.525 & 0.779 & 0.155 to 3.915 & 0.761 \\
\hline Positive Lymph nodes $(0 \mathrm{v} 1-3 \mathrm{v} 4-9 \mathrm{v} \geq 10)$ & 2.685 & 1.925 to 3.747 & $<0.01$ & 2.368 & 1.759 to 3.187 & $<0.01$ \\
\hline PD-L1 (positive v negative) & 0.302 & 0.127 to 0.721 & 0.007 & 0.451 & 0.211 to 0.963 & 0.04 \\
\hline Stromal TIL (0-10 v 11-40 v 41-100) & 0.418 & 0.092 to 1.891 & 0.257 & 1.249 & 0.337 to 4.632 & 0.74 \\
\hline TOTAL TIL (0-10 v 11-40 v 41-100) & 1.017 & 0.302 to 3.420 & 0.979 & 0.656 & 0.200 to 2.154 & 0.487 \\
\hline Stromal TIL $(<50 \mathrm{v} \geq 50(\mathrm{LPBC}))$ & 10.549 & 1.740 to 63.952 & 0.01 & 2.202 & 0.535 to 9.057 & 0.274 \\
\hline
\end{tabular}

Abbreviations: Programmed death ligand 1 (PD-L1) and stromal lymphocytic infiltrates (STILs) 
PD-L1 expression varied in different subtypes of breast cancer. A study had reported higher positive rates of PD-L1 expression in different types of tumor cells, including $20 \%$ in HER2-positive cells, 33\% in luminal subtype cells and over $59 \%$ in triple-negative breast cancer cells [19]. PD-L1 was expressed frequently in TNBC $(19 \%)$. In the single published research, PD-L1 was in a cohort of pure TNBC [20]. In our study, we showed that PD-L1 expression in TNBC occurred in $32.6 \%$ of all the patients. This different percentage of positive cases may be due to the differences in the antibody applied (5H1) in their study.

The relations of PD-L1 to a poor outcome have been shown in a number of researches on mixed cohorts. The PD-L1 expression was assessed as an index of adverse prognostic factor for other pernicious cancers, for example, NSCLC, renal cancer, melanoma, glioblastoma (GBM), colon cancer, neck and head squamous cell carcinoma and ovarian cancer [21-27]. However, according to current researches on NSCLC [28], PD-L1 is not related to adverse prognostic factor. Colorectal cancer [29] and melanoma [30] show that a favorable prognosis is related to PD-L1 expression. A prominent immune infiltrate has been shown in these researches whereas TILs have demonstrated to be related to $98 \%$ of PD-L1-positive tumors in melanoma [30] and PD-L1 was greatly related to in NSCLC [28].The expression of PD-L1might show a relation with a TIL-mediated antitumor inflammatory response, instead of being related to tumor immune evasion. In our current study, survival analysis showed that there was a trend for poorer overall survival for TNBC patients with low expression of PD-L1 conform to a current research (Schal-per et al. [31]) that evaluated mRNA. Also, statistical analysis has found that PD-L1 was strongly associated with TILs TNBC cohort. PD-L1 expression was not associated with age, Intra-tumoral TIL, Tumor size Positive Lymph Nodes, Stage, Chemotherapy, radiotherapy, and Neo-adjuvant chemotherapy. There was no strong correlation between the expression of PD-L1 and clinical behavior.

Despite the trend that PD-L1 expression seems to predict poorer prognosis in other cancers, the relation between PD-L1 and TNBC remains unclear. There already are argues over the topic. There already are argues over the topic. A study conducted by Tsang JYS et al. indicated a predict value of a poor prognosis on the existence of PD-L1, however, other studies showed no relation between PD-L1 expression and prognosis. Our results are opposite to the findings from the above paper. To our consideration, there might be two reasons leading to the difference between our study and others: 1) PD-L1 expression varied in different subtypes of breast cancer. OS of breast cancer patients includes all types in many studies, our study differed from Tsang's study because we focused only on TNBC patients. 2) Our result was similar to that of another study of PD-L1 on mRNA level, OS of TNBC patients with PD-L1 expressions was better than those without [32]. The differences between our result and those reporting connection of poorer prognosis and PD-L1 expression could potentially be due to how a positive PD-L1 cell was defined. The study conducted by Mittendorf et al. assessed PD-L1 expressions merely on tumor cell membrane of 105 TNBC patients. As stated above, solely existence of PD-L1 on cell membrane may not be related to prognosis, but co-evaluation of which on both cytoplasm and cytomembrane drew the conclusion that OS and DFS was significantly improved in PD-L1 high expression patients both in our and other study [33]. Meanwhile PD-L1 positive rate of Mittendorf's study was significantly lower than most studies on this subject [34], which may lead to bias in his results. A recent study also revealed that higher PD-L1 expression is related to higher PCR rate, which also indicated that patients were less likely to be suffering from poor prognosis [35].

In current days, various agents which target on the PD-1/PD-L1 system are at various phases of clinical exploration [36-39]. According to the finding of some researches, brisk immune infiltrate by TILs have been proved to associated with $98 \%$ of PD-L1-positive tumors in melanoma [36]; also, the TILs in NSCLC was correlated with the PD-L1 expression[27] . It can be predicted that PD-L1 expression might be related to a TIL-mediated antitumor inflammatory reaction, instead of relating to tumor immune escape as always.

This study found that PD-L1 expression and TILs in particular were biologically important in TNBC. According to recent findings by Tumeh et al. [40], TILs was probably basic predictor of treatment response. Our study might give support to clinical trials of this therapy in TNBC given the high expression of PD-L1 and higher STIL scores tumor compartments. Our data proved that PD-L1 was highly expressed in TNBC. The expression of PD-L1 was also correlated with the prevalence of TILs in TNBC. This study suggested that the potential of using PD-L1 was based on immunotherapy for the TNBC treatment. As an innovative and efficient treatment, immune checkpoint inhibitors are considered as a therapeutic method for RCC, NSCLC and melanoma which have been permitted by American FDA. Furthermore, clinical tests have been active in a wide scope of solid tumors, for instance, a small scale of ER-positive breast cancer and TNBC. A 
great amount of clinical tests in metastatic and neoadjuvant setting can determine the efficacy of immunotherapies and corresponding combinations in breast cancer.

\section{Acknowledgments}

We thank all patients, clinicians, and pathologists participating in the clinical studies.

\section{Ethical approval}

This article does not contain any studies with human participants or animals performed by any of the authors.

\section{Informed consent}

This article does not contain any studies with human participants.

\section{Competing Interests}

The authors have declared that no competing interest exists.

\section{References}

1. Siegel RL, Miller KD, Jemal A. Cancer statistics, 2015. CA: a cancer journal for clinicians. 2015; 65: 5-29.

2. Torre LA, Bray F, Siegel RL, Ferlay J, Lortet-Tieulent J, Jemal A. Global cancer statistics, 2012. CA: a cancer journal for clinicians. 2015; 65: 87-108.

3. Weigelt $B$, Peterse JL, van 't Veer LJ. Breast cancer metastasis: markers and models. Nature reviews Cancer. 2005; 5: 591-602.

4. Isakoff SJ. Triple-Negative Breast Cancer Role of Specific Chemotherapy Agents. Cancer J. 2010; 16: 53-61.

5. Greenwald RJ, Freeman GJ, Sharpe AH. The B7 family revisited. Annual review of immunology. 2005; 23: 515-48.

6. Zou WP, Chen LP. Inhibitory B7-family molecules in the tumour microenvironment. Nat Rev Immunol. 2008; 8: 467-77.

7. Freeman GJ, Long AJ, Iwai $Y$, Bourque $K$, Chernova T, Nishimura H, et al. Engagement of the PD-1 immunoinhibitory receptor by a novel B7 family member leads to negative regulation of lymphocyte activation. J Exp Med. 2000; 192: 1027-34.

8. Ishida Y, Agata Y, Shibahara K, Honjo T. Induced expression of PD-1, a novel member of the immunoglobulin gene superfamily, upon programmed cell death. The EMBO journal. 1992; 11: 3887-95.

9. Cho YA, Yoon HJ, Lee JI, Hong SP, Hong SD. Relationship between the expressions of PD-L1 and tumor-infiltrating lymphocytes in oral squamous cell carcinoma. Oral Oncol. 2011; 47: 1148-53

10. Muenst S, Soysal SD, Gao F, Obermann EC, Oertli D, Gillanders WE. The presence of programmed death 1 (PD-1)-positive tumor-infiltrating lymphocytes is associated with poor prognosis in human breast cancer. Breast Cancer Res Tr. 2013; 139: 667-76.

11. Berghoff AS, Kiesel B, Widhalm G, Rajky O, Ricken G, Wohrer A, et al. Programmed death ligand 1 expression and tumor-infiltrating lymphocytes in glioblastoma. Neuro-Oncology. 2015; 17: 1064-75.

12. D'Angelo SP, Shoushtari AN, Agaram NP, Kuk D, Qin LX, Carvajal RD, et al. Prevalence of tumor-infiltrating lymphocytes and PD-L1 expression in the soft tissue sarcoma microenvironment. Hum Pathol. 2015; 46: 357-65.

13. Berghoff AS, Ricken G, Widhalm G, Rajky O, Dieckmann K, Birner P, et al. Tumour-infiltrating lymphocytes and expression of programmed death ligand 1 (PD-L1) in melanoma brain metastases. Histopathology. 2015; 66: 289-99.

14. Kakavand $\mathrm{H}$, Wilmott JS, Menzies AM, Vilain R, Haydu LE, Yearley JH, et al. PD-L1 Expression and Tumor-Infiltrating Lymphocytes Define Different Subsets of MAPK Inhibitor-Treated Melanoma Patients. Clin Cancer Res. 2015; 21: 3140-8.

15. Sun SY, Fei XC, Mao Y, Wang XM, Garfield DH, Huang O, et al. PD-1(+) immune cell infiltration inversely correlates with survival of operable breast cancer patients. Cancer Immunol Immun. 2014; 63: 395-406.

16. Pardoll DM. The blockade of immune checkpoints in cancer immunotherapy. Nature Reviews Cancer. 2012; 12: 252-64

17. Taube JM, Klein A, Brahmer JR, Xu H, Pan X, Kim J, et al. Association of PD-1, PD-1 ligands, and other features of the tumor immune microenvironment with response to anti-PD-1 therapy. J Invest Dermatol. 2014; 134: S92-S.

18. Loi S. Host Antitumor Immunity Plays a Role in the Survival of Patients With Newly Diagnosed Triple-Negative Breast Cancer. J Clin Oncol. 2014; 32: 2935-+.
19. Gatalica Z, Snyder C, Maney T, Ghazalpour A, Holterman DA, Xiao NQ, et al. Programmed Cell Death 1 (PD-1) and Its Ligand (PD-L1) in Common Cancers and Their Correlation with Molecular Cancer Type. Cancer Epidem Biomar. 2014; 23: 2965-70.

20. Mittendorf EA, Philips AV, Meric-Bernstam F, Qiao N, Wu Y, Harrington S, et al. PD-L1 Expression in Triple-Negative Breast Cancer. Cancer Immunol Res. 2014; 2: 361-70.

21. Yamazaki T, Akiba H, Iwai H, Matsuda H, Aoki M, Tanno Y, et al. Expression of programmed death 1 ligands by murine T cells and APC. J Immunol. 2002; 169: 5538-45.

22. Parsa AT, Waldron JS, Panner A, Crane CA, Parney IF, Barry JJ, et al. Loss of tumor suppressor PTEN function increases B7-H1 expression and immunoresistance in glioma. Nat Med. 2007; 13: 84-8.

23. Hamanishi J, Mandai M, Iwasaki M, Okazaki T, Tanaka Y, Yamaguchi K, et al. Programmed cell death 1 ligand 1 and tumor-infiltrating CD8+ T lymphocytes are prognostic factors of human ovarian cancer. Proceedings of the National Academy of Sciences of the United States of America. 2007; 104: 3360-5.

24. Thompson RH, Gillettt MD, Cheville JC, Lohse CM, Dong HD, Webster WS, et al. Costimulatory B7-H1 in renal cell carcinoma patients: Indicator of tumor aggressiveness and potential therapeutic target. Proceedings of the National Academy of Sciences of the United States of America. 2004; 101: 17174-9.

25. Strome SE, Dong HD, Tamura H, Voss SG, Flies DB, Tamada K, et al. B7-H1 blockade augments adoptive T-cell immunotherapy for squamous cell carcinoma. Cancer Res. 2003; 63: 6501-5.

26. Ohigashi $Y$, Sho M, Yamada $Y$, Tsurui $Y$, Hamada K, Ikeda N, et al. Clinical significance of programmed death-1 ligand-1 and programmed death-1 ligand-2 expression in human esophageal cancer. Clin Cancer Res. 2005; 11: 2947-53.

27. Konishi J, Yamazaki K, Azuma M, Kinoshita I, Dosaka-Akita H, Nishimura M. B7-h1 expression on non-small cell lung cancer cells and its relationship with tumor-infiltrating lymphocytes and their PD-1 expression. Clin Cancer Res. 2004; 10: 5094-100.

28. Velcheti V, Schalper KA, Carvajal DE, Anagnostou VK, Syrigos KN, Sznol M, et al. Programmed death ligand-1 expression in non-small cell lung cancer. Lab Invest. 2014; 94: 107-16.

29. Droeser RA, Hirt C, Viehl CT, Frey DM, Nebiker C, Huber X, et al. Clinical impact of programmed cell death ligand 1 expression in colorectal cancer. Eur J Cancer. 2013; 49: 2233-42.

30. Taube JM, Anders RA, Young GD, Xu HY, Sharma R, McMiller TL, et al. Colocalization of Inflammatory Response with B7-H1 Expression in Human Melanocytic Lesions Supports an Adaptive Resistance Mechanism of Immune Escape. Sci Transl Med. 2012; 4

31. Schalper KA, Velcheti V, Carvajal D, Wimberly H, Brown J, Pusztai L, et al. In Situ Tumor PD-L1 mRNA Expression Is Associated with Increased TILs and Better Outcome in Breast Carcinomas. Clin Cancer Res. 2014; 20: 2773-82.

32. Dong H, Strome SE, Salomao DR, Tamura H, Hirano F, Flies DB, et al. Tumor-associated B7-H1 promotes T-cell apoptosis: a potential mechanism of immune evasion. Nature medicine. 2002; 8: 793-800.

33. Beckers R K SCI, Vilain R, Madore J et al. Programmed death ligand 1 expression in triple-negative breast cancer is associated with tumour-infiltrating lymphocytes and improved outcome. Histopathology. 2016: 2016,69 (1) $\cdot 25$.

34. Mittendorf EA, Philips AV, Meric-Bernstam F, Qiao N, Wu Y, Harrington S, et al. PD-L1 expression in triple-negative breast cancer. Cancer immunology research. 2014; 2 : 361-70.

35. V Pelekanouet al. Effects of neoadjuvant chemotherapy (NAC) on tumor infiltrating lymphocytes (TIL) and PD-L1 expression in the SWOG S0800 clinical trial. ASCO 2017.

36. Hamid O, Robert C, Daud A, Hodi FS, Hwu WJ, Kefford R, et al. Safety and Tumor Responses with Lambrolizumab (Anti-PD-1) in Melanoma. New Engl J Med. 2013; 369: 134-44

37. Topalian SL, Hodi FS, Brahmer JR, Gettinger SN, Smith DC, McDermott DF, et al. Safety, Activity, and Immune Correlates of Anti-PD-1 Antibody in Cancer. New Engl J Med. 2012; 366: 2443-54

38. Brahmer JR, Drake CG, Wollner I, Powderly JD, Picus J, Sharfman WH, et al. Phase I Study of Single-Agent Anti-Programmed Death-1 (MDX-1106) in Refractory Solid Tumors: Safety, Clinical Activity, Pharmacodynamics, and Immunologic Correlates. J Clin Oncol. 2010; 28: 3167-75.

39. Brahmer JR, Tykodi SS, Chow LQM, Hwu WJ, Topalian SL, Hwu P, et al. Safety and Activity of Anti-PD-L1 Antibody in Patients with Advanced Cancer. New Engl J Med. 2012; 366: 2455-65.

40. Tumeh PC, Harview CL, Yearley JH, Shintaku IP, Taylor EJM, Robert L, et al. PD-1 blockade induces responses by inhibiting adaptive immune resistance. Nature. 2014; 515: 568-+ 